\title{
Sleep-Dependent Changes in the Coupling Between Heart Period and Arterial Pressure in Newborn Lambs
}

\author{
ALESSANDRO SILVANI, VALENTINA ASTI, TIJANA BOJIC, VERA FERRARI, \\ CARLO FRANZINI, PIERLUIGI LENZI, DANIEL A. GRANT, ADRIAN M. WALKER, AND \\ GIOVANNA ZOCCOLI \\ Dipartimento di Fisiologia Umana e Generale [A.S., V.A., T.B., V.F., C.F., P.L., G.Z.], Università di Bologna, \\ 40127 Bologna, Italy; and Ritchie Centre for Baby Health Research [D.A.G., A.M.W.], Monash University, \\ 3168 Clayton, Victoria, Australia
}

\begin{abstract}
This study assessed whether sleep-dependent changes in the relationship between heart period (HP) and mean arterial pressure (MAP) occur in newborn life. Electrodes for electrocorticographic, electromyographic, and electrooculographic monitoring and an arterial catheter for blood pressure recordings were implanted in 11 newborn lambs. HP and MAP beat-to-beat values were computed from 120 -s blood pressure recordings during quiet wakefulness, active sleep, and quiet sleep. For each recording, the time shift at which the maximum of the HP versus MAP cross-correlation function was attained was identified. For each lamb and wake-sleep state, an average correlation coefficient was then computed corresponding to the median value of such time shifts. The maximum of the cross-correlation function was attained with HP lagging behind MAP. The corresponding mean correlation coefficient was significantly higher in quiet sleep $(0.51 \pm 0.05)$ than either in quiet wakefulness $(0.31 \pm$
\end{abstract}

Autonomic and peripheral circulatory functions are strongly affected by wake-sleep states (1). On the basis of studies of thermoregulation and of respiratory and circulatory control, it was suggested that in quiet sleep, homeostasis is tightly maintained by closed-loop control mechanisms, whereas in active sleep, an open-loop, nonhomeostatic mode of regulation prevails (2).

The regulation of systemic arterial pressure plays an essential role in homeostasis, as it permits coupling of blood flow to tissue metabolic needs while avoiding excessive rises in microvessel transmural pressure. Central autonomic commands,

Received February 11, 2004; accepted July 6, 2004.

Correspondence: Giovanna Zoccoli, M.D., Dipartimento di Fisiologia Umana e Generale, Università di Bologna, Piazza di Porta San Donato, 240127 Bologna, Italy; e-mail giovanna.zoccoli@unibo.it

This study was supported by the National Health and Medical Research Council of Australia (to A.M.W. and D.A.G.); the Department of Industry, Science, and Tourism of Australia (to A.M.W. and D.A.G.); and the Monash University Research Fund (to D.A.G.).

DOI: 10.1203/01.PDR.0000148065.32413.B0
$0.05)$ or in active sleep $(0.29 \pm 0.03)$. Sleep-related differences in the correlation between HP and MAP were maintained after $\mathrm{HP}$ and MAP data were low-pass filtered at $0.3 \mathrm{~Hz}$ to remove their fast ventilatory oscillations. In conclusion, data indicate that the relationship between spontaneous fluctuations in HP and those in MAP is sleep-state dependent in newborn lambs. A positive HP versus MAP correlation with HP lagging behind MAP is consistent with baroreflex control of HP. Heart rhythm thus may be more tightly controlled by the baroreceptor reflex and less dependent on central autonomic commands in quiet sleep than either in quiet wakefulness or in active sleep. (Pediatr Res 57: 108-114, 2005)

HP, heart period

Abbreviations

MAP, mean arterial pressure

in supporting an actual or expected behavior [e.g. exercise (3), defense reaction (4)], may induce parallel changes in heart rate and in systemic vascular resistance. In adult rats, baroreflex control of heart rate prevails in quiet sleep, whereas central commands prevail in active sleep $(5,6)$. Although effects of central autonomic commands on heart rate and arterial pressure are similar in wakefulness and in active sleep, the cause of such commands is uncertain in the latter state. Thus, central autonomic commands that occur in active sleep give rise to regulatory disturbances rather than to anticipatory regulation, as it occurs during wakefulness. It is unclear whether these conclusions apply to animals during early postnatal development, whose cardiovascular regulation undergoes functional maturation and differs in many respects from that of older animals (7-12).

The aim of our study was to assess whether the relationship between heart period (HP) and arterial pressure changes during sleep in newborn lambs. We analyzed the relationship between HP and mean arterial pressure (MAP) in the time domain by 
computing a cross-correlation function between spontaneous fluctuations in the two variables. Linear correlation has been proved to be a powerful tool to evaluate the balance of central and baroreflex control of HP in adult rats (6). The crosscorrelation function allows a more complete description of the system under study, as it yields information on the correlation between two signals as a function of the time shift, i.e. positive or negative time lag, between the signals (13). Thus, the maximum value reached by the cross-correlation function can quantify the strength of the coupling between HP and arterial pressure. The time shift observed, however, informs on the time relationship between changes in arterial pressure and changes in HP.

Sleep-related differences in cardiovascular regulation are potentially of importance in newborn life, when total sleep duration and the duration of active sleep are at a lifetime maximum (8). Moreover, sudden infant death syndrome has been associated with autonomic derangements (14), which may be especially prominent during specific sleep states (15). Knowledge of physiologic sleep-related changes in cardiovascular regulation thus may help in the early detection of pathologic derangements in autonomic cardiovascular control.

\section{METHODS}

Eleven newborn lambs (Merino/Border-Leicester cross) were housed within $24 \mathrm{~h}$ of birth in a Plexiglas cage. Once feeding independently (lamb milk replacer: Veanavite; Shepparton, Australia) and gaining weight normally, each lamb was surgically prepared for chronic study. All procedures were performed in accordance with the guidelines of the Australian Code of Practice for the Care and Use of Animals for Scientific Purposes established by the National Health and Medical Research Council of Australia and were approved by the Monash Medical Centre Committee on Ethics in Animal Experimentation.

Surgery and experimental procedures. Each lamb was anesthetized (halothane $1-2 \%$, nitrous oxide $60 \%$, balance oxygen) and instrumented for study with the use of sterile surgical techniques under artificial ventilation. A nonocclusive saline-filled catheter $(0.86$ and $1.52 \mathrm{~mm}$ in inner and outer diameter, respectively) was inserted in the femoral artery for arterial blood pressure monitoring and blood sampling. Pairs of Teflon-coated stainless steel wires were implanted on the parietal cortex $1 \mathrm{~cm}$ anterior and $1 \mathrm{~cm}$ lateral to lambda (electrocorticogram), at the inner and outer canthus of the left eye (electrooculogram), and in the dorsal musculature of the neck (nuchal electromyogram).

Measurements. After a minimum of $72 \mathrm{~h}$ of postoperative recovery, the lambs were studied for 1-3 d, at the age of $18 \pm 2 \mathrm{~d}$ (mean \pm SEM). During the study periods, the lambs' cages were partitioned to prevent the lambs from turning around while allowing complete freedom to move forward and backward and to stand up and lie down. The lambs did not show any sign of discomfort during the recordings, such as struggles to turn around despite the cage divider. Food was available ad libitum throughout the study, and room temperature was maintained between 22 and $25^{\circ} \mathrm{C}$. Electrophysiologic signals were amplified and filtered with a signal conditioner (Cyberamp 380; Axon Instruments, Foster City, CA) at $0.3-80 \mathrm{~Hz}, 0.3-80 \mathrm{~Hz}$, and $30-80 \mathrm{~Hz}$ for electrocorticogram, electrooculogram, and nuchal electromyogram, respectively. The arterial catheter was connected to a calibrated strain-gauge manometer (Cobe CDX III; Cobe Laboratories, Lakewood, CO), which in turn was connected to the signal conditioner. Arterial pressure was referenced to the midthoracic level when the lambs were lying down, low-pass filtered at 100 $\mathrm{Hz}$, and, along with the electrophysiologic signals, stored on a computer using an analog-digital converting board (model 4801A; ADAC, Woburn, MA) and acquisition software (CVSOFT Data Acquisition and Analysis Software; Odessa Computer Systems, Calgary, Alberta, Canada).

Data analysis. After the study was completed, the stored signals were reviewed to reject artefacts including movement artefacts. Data sequences
$120 \mathrm{~s}$ long sampled at a rate of $200 \mathrm{~Hz}$ were selected during stable states of quiet wakefulness, quiet sleep, and active sleep. Sequences were selected no less than $5 \mathrm{~s}$ after the occurrence of artefacts and only when the polygraphic recordings indicated the stability of the behavioral state throughout the whole data segment extracted. Thus, transient events such as sleep-state transitions, microarousals, and spontaneous awakenings were excluded from analysis. The 120 -s duration was chosen to ensure both an adequate length of the sequence and the absence of artefacts, sleep-state transitions, and arousals in the same sequence.

The scoring of the wake-sleep state was performed manually on the basis of behavioral and polygraphic criteria. In quiet sleep, the electrocorticogram displayed a pattern of high-voltage (i.e. the signal amplitude seemed to be at least $50 \%$ of the maximum signal amplitude observed throughout the data file (16)) and low-frequency (i.e. large predominance of the 1- to 4-Hz frequency band (16)) activity, eye movements were absent, and nuchal electromyographic tone was reduced compared with that in quiet wakefulness. Quiet wakefulness was identified when the lambs were lying down, when the electrocorticogram displayed a pattern of lower-voltage and higher-frequency activity with respect to that in quiet sleep, and when eye movements and nuchal electromyographic tone were present. During active sleep, the electrocorticogram displayed a pattern of lower-voltage and higher-frequency activity with respect to that in quiet sleep, rapid eye movements were present, and nuchal electromyographic tone was absent.

To increase the reliability of the computed estimates, for each lamb, only wake-sleep states in which it was possible to collect three or more artefact-free sequences were considered for analysis. No artefact-free sequence could be obtained in quiet wakefulness in one lamb and in active sleep in another lamb. In three other lambs, we obtained only one, two, and two artefact-free sequences in active sleep, respectively. Analysis thus was performed on a total of 163 sequences collected during quiet wakefulness, 373 during quiet sleep, and 198 during active sleep. Signal analysis was performed with the software package MATLAB and its Signal Processing Toolbox (The MathWorks, Inc., Natick, MA).

HP was automatically determined from the arterial pressure signal as the time interval between the beginnings of successive systolic upstrokes, and accuracy of the determination was ensured by manual editing of all of the tracings. MAP was computed as the average arterial pressure during each heart period. Averages for HP and MAP were calculated over each 120-s sequence. Beat-to-beat HP and MAP data were resampled at $4 \mathrm{~Hz}$ by linear interpolation to yield data as a function of time rather than of cardiac cycle, and their mean values were subtracted. The HP versus MAP cross-correlation function was then computed for each 120 -s sequence between time shifts from $-12.5 \mathrm{~s}$ to 12.5 s. With our computational procedure, the HP versus MAP crosscorrelation function at a negative time shift reflects the correlation between MAP values and HP values that follow those of MAP by the modulus of the time shift. The cross-correlation function was computed as the average crosscorrelation over five data subsets $60 \mathrm{~s}$ long overlapped by $75 \%$. Values of the cross-correlation functions were normalized so that the autocorrelations for HP and MAP at zero time shift were identically 1.0.

The time shift that corresponded to the maximal positive value of the cross-correlation function between HP and MAP was identified for each 120-s sequence. The median value of that time shift was computed for each lamb and wake-sleep state. For each lamb and wake-sleep state, the mean value of the cross-correlation function was subsequently calculated, corresponding to the time shift previously determined in that lamb and state.

In previous work (17), we showed that variability of cerebral perfusion pressure in the lamb has a similar frequency distribution in all behavioral states, with a minimum at $\sim 0.3 \mathrm{~Hz}$ and a high-frequency peak at frequencies between 0.3 and $1.2 \mathrm{~Hz}$, comparable with values of breathing rate reported in the lamb (18). To assess whether the cross-correlation estimates computed were dependent on fast, presumably ventilatory oscillations in MAP, we repeated the cross-correlation analysis after low-pass filtering HP and MAP data at $0.3 \mathrm{~Hz}$ by means of a six-pole Chebyshev type I digital filter with band-pass ripple limited to $0.5 \mathrm{~dB}$. HP and MAP data sequences were first filtered in the forward direction, then reversed and run back through the filter to obtain low-pass filtered data with zero phase distortion.

To gain further insight into cardiovascular regulation in active sleep, we also analyzed HP and MAP during the phasic hypertensive events [MAP surges $(19,20)]$ that occur spontaneously in this state. A total of 95 sequences 
of beat-to-beat HP and MAP data composing an increase in MAP $>15 \%$ was identified in active sleep on seven lambs. HP and MAP data were divided by their baseline values, i.e. by their respective mean values over the 20 beats preceding the beginning of the MAP surge. A coherent averaging of the data was then performed for each lamb by aligning the data sequences temporally with the beginning of the MAP surges (13).

Statistical analysis. Mean values for each wake-sleep state were calculated for each animal. One-sample $t$ tests were applied to detect significant differences from time 0 shifts in the cross-correlation analysis and to detect significant differences from 1 of the ratios between HP values and their baseline value in the analysis of MAP surges. When significant differences of time shifts and of values of the cross-correlation function among wake-sleep states were detected by the Friedman test, the significance of differences between wake-sleep states was assessed by Wilcoxon test. Statistical testing was performed using standard procedures (SPSS, http://www.spss.com) with a $p<0.05$ considered to be statistically significant. Data are means \pm SEM in the text, table, and figures, with $n$ indicating the number of animals.

\section{RESULTS}

Arterial blood gases and $\mathrm{pH}$ were similar to those previously recorded in healthy lambs $(17,18)(\mathrm{pH}=7.429 \pm 0.007$, arterial $\mathrm{Po}_{2}=103.2 \pm 3.2 \mathrm{~mm} \mathrm{Hg}$, arterial $\mathrm{O}_{2}$ saturation $=$ $95.6 \pm 0.9 \%$, arterial $\mathrm{PCO}_{2}=41.7 \pm 1.2 \mathrm{~mm} \mathrm{Hg}$, hemoglobin concentration $=8.1 \pm 0.5 \mathrm{~g} / \mathrm{dL}$, and base excess $=2.9 \pm 0.9$ $\mathrm{mM})$.

Mean values of HP were $380 \pm 18,365 \pm 16$, and $402 \pm 28$ $\mathrm{ms}$ in quiet wakefulness, quiet sleep, and active sleep, respectively. Mean values of MAP were 72.9 $\pm 1.7,72.9 \pm 1.4$, and $73.2 \pm 2.7 \mathrm{~mm} \mathrm{Hg}$ in quiet wakefulness, quiet sleep, and active sleep, respectively. MAP did not differ significantly in mean value among wake-sleep states, but a difference among behavioral states did exist in HP, which was significantly higher in active sleep than in quiet sleep, in agreement with previous findings $(18,19)$.

HP versus MAP cross-correlation. Both on unfiltered data (Fig. 1) and on their low-frequency fluctuations (Fig. 2), the peaks of the average HP versus MAP cross-correlation functions were evident at a negative time shift, indicating that the highest positive correlation between HP and MAP ensued with HP changes lagging behind MAP changes.

Time shifts between HP and MAP corresponding to the maximal positive value of the HP versus MAP crosscorrelation function are reported in Table 1. Both on unfiltered data and on their low-frequency fluctuations, time shifts between HP and MAP were negative and significantly different from $0(p<0.005)$ and were not significantly different among wake-sleep states. In quiet sleep, the time lag of HP versus MAP computed on low-pass filtered data was higher than the corresponding time lag computed on unfiltered data $(p<0.05)$.

On unfiltered data, mean values of the HP versus MAP cross-correlation functions corresponding to the time shifts that maximized the HP versus MAP correlation were $0.31 \pm 0.05$, $0.51 \pm 0.05$, and $0.29 \pm 0.03$ in quiet wakefulness, quiet sleep, and active sleep, respectively (Fig. 3). Mean values of the HP versus MAP cross-correlation functions computed on low-pass filtered data and corresponding to the time shifts that maximized the HP versus MAP correlation at low frequencies were $0.35 \pm 0.05,0.61 \pm 0.04$, and $0.32 \pm 0.03$ in quiet wakefulness, quiet sleep, and active sleep, respectively (Fig. 4). Both on unfiltered data and on their low-frequency fluctuations, the
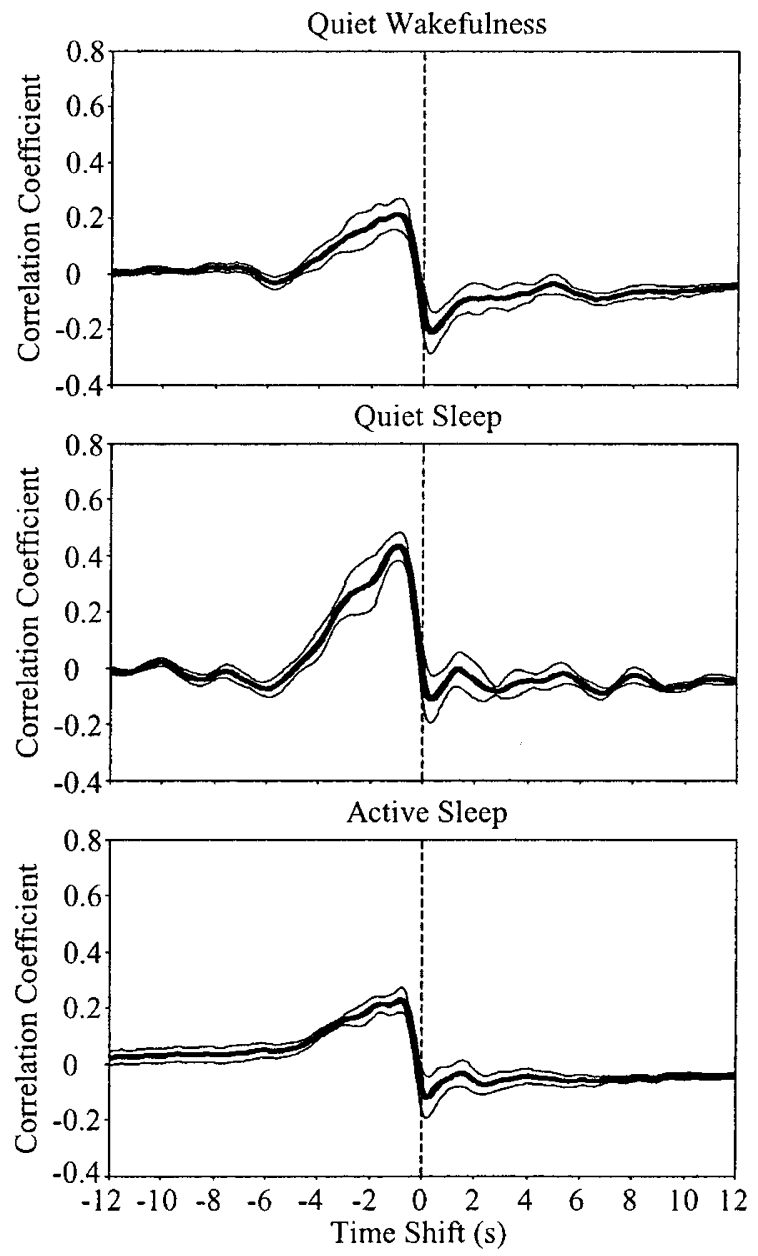

Figure 1. Cross-correlation functions between HP and MAP computed on unfiltered data. Bold lines represent mean values of the cross-correlation functions; thin lines represent mean values \pm SEM. In all states, a peak in the crosscorrelation function was evident at a negative time shift, indicating that HP was positively correlated with preceding values of MAP. The correlation coefficient at the peak of the cross-correlation function was highest in quiet sleep. $n=10$ in quiet wakefulness; $n=11$ in quiet sleep; $n=7$ in active sleep.

HP versus MAP correlation coefficient was significantly higher in quiet sleep than either in quiet wakefulness or in active sleep $(p<0.05)$. In quiet sleep, the HP versus MAP correlation coefficient computed on low-pass filtered data was higher than the corresponding correlation coefficient computed on unfiltered data $(p<0.05)$. In agreement with results of the timedomain data analysis, visual analysis of single data sequences revealed that, particularly in quiet sleep, changes in HP followed a pattern similar and parallel to that of previous changes in MAP; the similarity between patterns of changes in HP and in MAP was readily evident on low-pass filtered data (Fig. 5).

HP changes during phasic MAP surges in active sleep. Analysis of phasic cardiovascular events in active sleep revealed that transient decreases in HP occurred concomitantly with the beginnings of the MAP surges and were followed by rises in HP above control values during the course of the MAP surges (Fig. 6). The decrease in HP started in the first cardiac cycle after the beginning of the MAP surge $(p<0.01)$, and the mean value of HP over nine cardiac cycles after the onset of the MAP surge was reduced with respect to its control value $(p<$ 

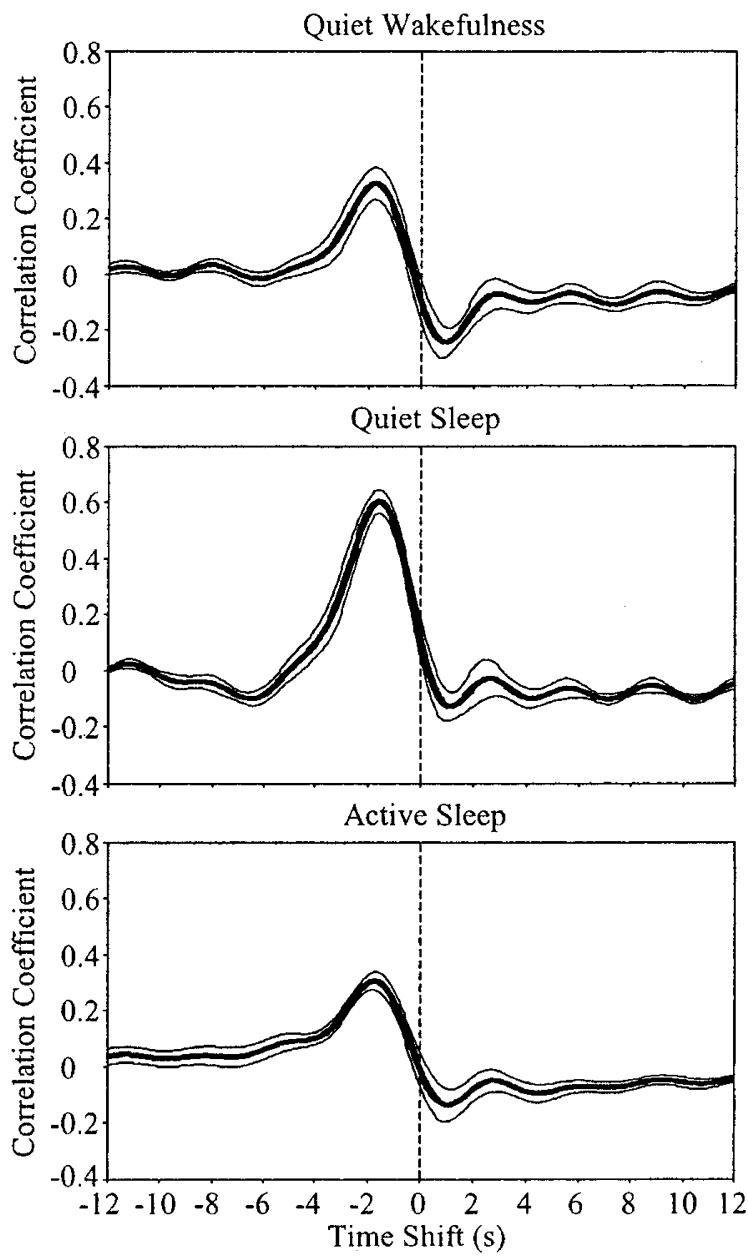

Figure 2. Cross-correlation functions between HP and MAP computed on data low-pass filtered at $0.3 \mathrm{~Hz}$. Bold lines represent mean values of the cross-correlation functions computed on low-pass filtered data; thin lines represent mean values \pm SEM. Cross-correlation functions computed on low-pass filtered data shared the same features of those computed on unfiltered data, in that peaks of the functions were evident at a negative time shift and the value at the peak was highest in quiet sleep. $n=10$ in quiet wakefulness; $n=$ 11 in quiet sleep; $n=7$ in active sleep.

Table 1. Time shifts between HP and MAP corresponding to the highest positive correlation between the variables

\begin{tabular}{lclc}
\hline & Quiet wakefulness & Quiet sleep & Active sleep \\
\hline TS & $-1.7 \pm 0.2$ & $-1.4 \pm 0.2$ & $-1.6 \pm 0.2$ \\
TS LF & $-2.1 \pm 0.2$ & $-1.7 \pm 0.1^{*}$ & $-1.8 \pm 0.2$ \\
\hline
\end{tabular}

Values represent mean \pm SEM. TS, time shift (s) between HP and MAP corresponding to the highest positive correlation between the variables; TS LF, TS computed on data low-pass filtered at $0.3 \mathrm{~Hz} . n=10$ in quiet wakefulness; $n=11$ in quiet sleep; $n=7$ in active sleep.

$* p<0.05$ vs TS in the same state.

0.005). Conversely, the mean value of HP over subsequent cardiac cycles was increased with respect to control values $(p$ $<0.01)$.

\section{DISCUSSION}

In newborn lambs, we assessed whether sleep-dependent changes occur in the cross-correlation function between spontaneous fluctuations in HP and those in MAP. The correlation

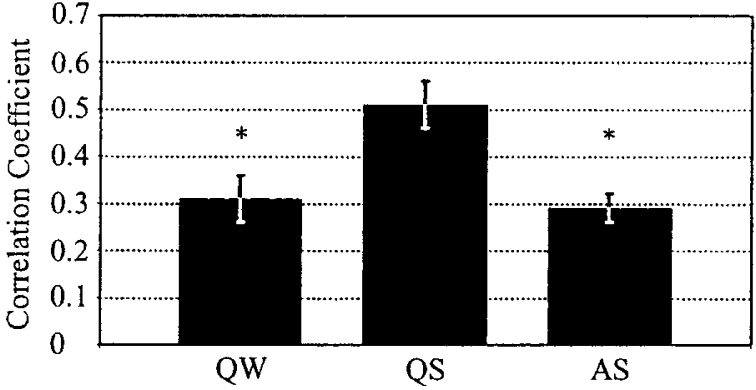

Figure 3. Correlation coefficient between HP and MAP at the time lag corresponding to maximal correlation, computed on unfiltered data. Mean values are shown. Error bars represent \pm SEM. QW, quiet wakefulness; QS, quiet sleep; AS, active sleep. The correlation coefficient was highest in QS. * $p$ $<0.05 v s$ value in QS. $n=10$ in QW; $n=11$ in QS; $n=7$ in AS.

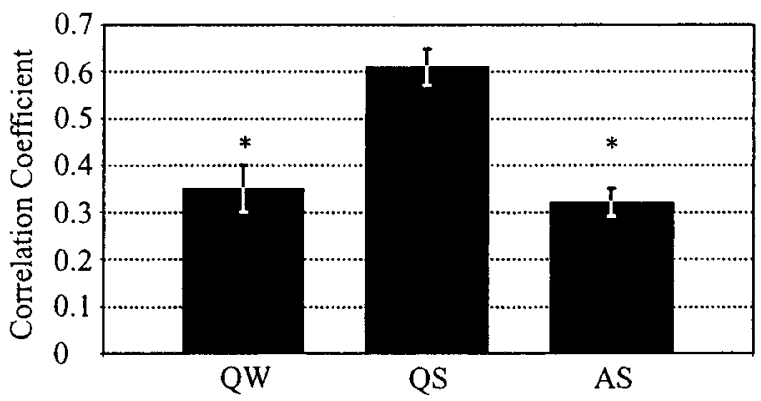

Figure 4. Correlation coefficient between HP and MAP at the time lag corresponding to maximal correlation, computed on data low-pass filtered at $0.3 \mathrm{~Hz}$. Mean values are shown. Error bars represent \pm SEM. QW, quiet wakefulness; QS, quiet sleep; AS, active sleep. On low-pass filtered data, the correlation coefficient in QS was $>70 \%$ higher than either in QW or in AS, indicating that a difference in the correlation coefficient among wake-sleep states existed irrespective of fast fluctuations in HP and MAP. ${ }^{*} p<0.05 v s$ value in QS. $n=10$ in $\mathrm{QW} ; n=11$ in $\mathrm{QS} ; n=7$ in $\mathrm{AS}$.

between HP and previous values of MAP was positive and was $>60 \%$ higher in quiet sleep than in the other states, suggesting that heart rhythm is more tightly controlled by the baroreceptor reflex and less dependent on central autonomic commands in quiet sleep than either in quiet wakefulness or in active sleep. During phasic hypertensive events in active sleep, central autonomic commands on HP displayed a time course different from that of baroreflex control: HP decreased almost simultaneously with the rise in MAP, then increased consistently with baroreflex control.

Cardiovascular control in newborn animals undergoes functional maturation (10). Basal cardiac output per unit body weight $(7,8)$ and heart rate $(9)$ are high with respect to values in older animals, whereas arterial blood pressure is low $(7,21)$. The arterial baroreflex is effective and reset to lower levels of arterial pressure, whereas cardiopulmonary reflexes are impaired (12). The impact of the sympathetic nervous system on heart rate variability decreases after the first month of life in lambs (9) and human infants (11). Finally, the responsiveness of peripheral vessels to adrenergic agonists is lower in newborn than in adult lambs (7). We performed our study in newborn lambs, a model widely used in the study of sleep and cardiovascular physiology during early postnatal development. Further research is needed to investigate the extent to which the 

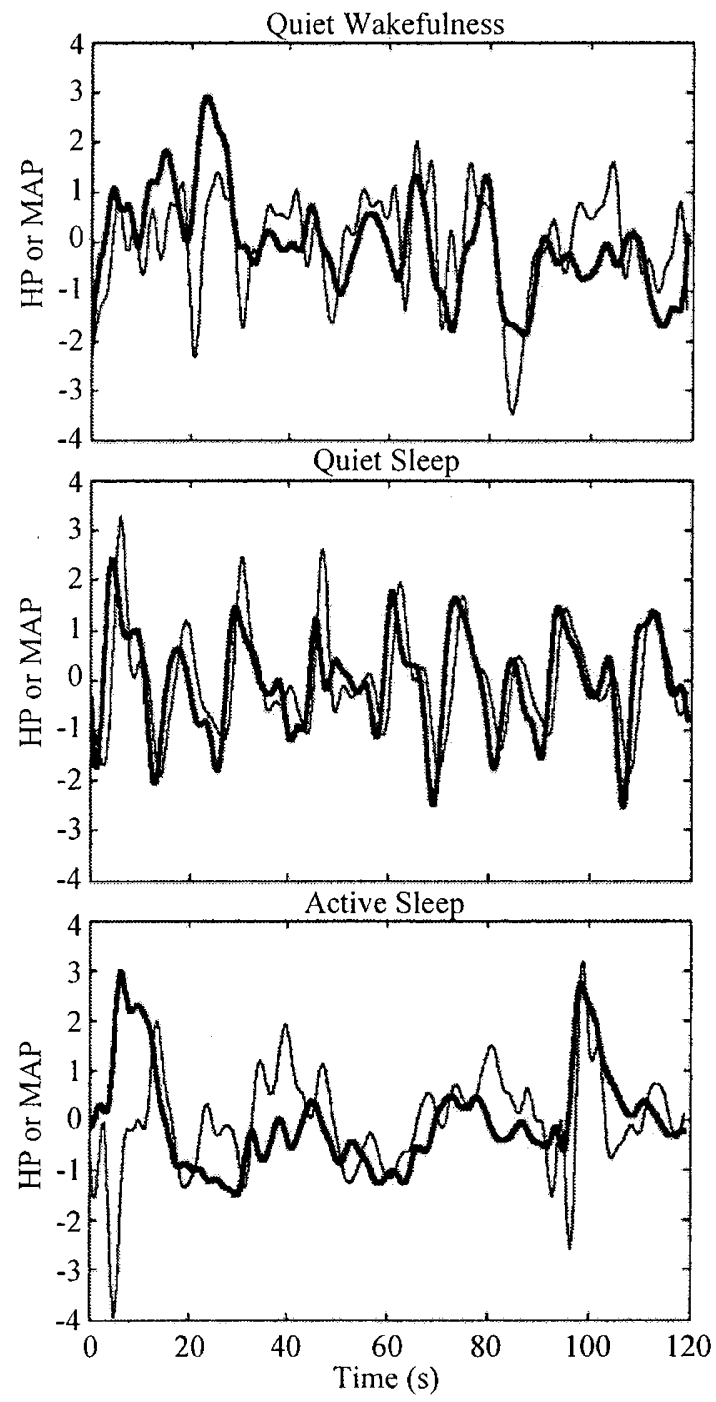

Figure 5. Representative sequences of low-frequency fluctuations in HP and MAP. Thin line represents HP; bold line represents MAP. Values are in standardized units. Beat-to-beat data obtained in one lamb were resampled at $4 \mathrm{~Hz}$ and low-pass filtered at $0.3 \mathrm{~Hz}$. $\mathrm{HP}$ was coupled to previous changes in MAP, with the tightest coupling occurring in quiet sleep.

features of cardiovascular control that we described in newborns differ from those in adult animals.

The study of spontaneous fluctuations in HP and MAP informs on cardiovascular regulation around its working point, as such fluctuations are a function of autonomic activity and of the properties (time delay, frequency response) of cardiovascular control mechanisms (22). HP and MAP are related by a closed loop. HP has an impact on cardiac output, and hence on MAP, MAP feeds back on HP via the baroreceptor reflex. The mechanical effect of HP on MAP entails a negative correlation between the variables, with changes in HP preceding or concomitant with those in MAP (23). Thus, the positive HP versus MAP correlation that we found with changes in HP following changes in MAP is not explained by the mechanical effect of HP on MAP but rather may reflect baroreflex coupling between the variables.

The relationship between HP and MAP changes is a function of the frequency range analyzed. At breathing rate, fluctuations
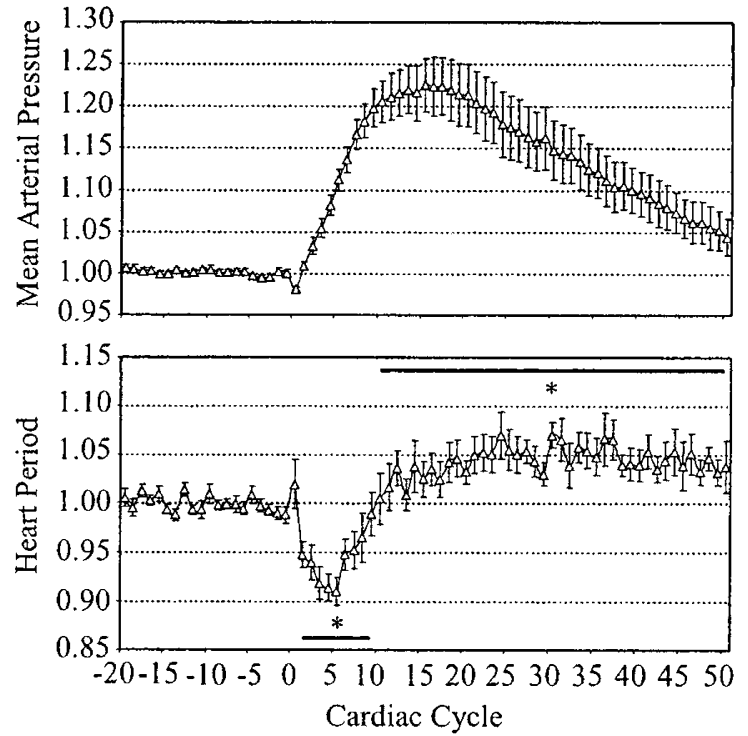

Figure 6. Beat-to-beat changes in HP and MAP during phasic blood pressure surges in active sleep. Beat-to-beat values of HP and MAP were divided by their baseline values and averaged with the cardiac cycles corresponding with the onset of the MAP surges coinciding. Mean values \pm SEM are shown. HP started to decrease at the onset of the surge; an increase in HP is evident later in the course of the MAP surge, consistent with baroreflex control. ${ }^{*} p<0.05$ for the difference between HP (average value over the cardiac cycles identified by horizontal lines) and its baseline value. $n=7$.

in HP are determined not only by baroreflex buffering of MAP variability but also by central coupling of respiratory drive to cardiac vagal motor neurons (22). We repeated the crosscorrelation analysis after low-pass filtering HP and MAP data at $0.3 \mathrm{~Hz}$ to determine whether any sleep-related difference in the relationship between HP and MAP was altered by the contribution of fast ventilatory fluctuations. Results obtained on low-pass filtered data (Fig. 2) were similar to those obtained on unfiltered data (Fig. 1), indicating that the main features of the HP versus MAP relationship identified by the present analysis were independent of fluctuations in the two variables at a frequency higher than $0.3 \mathrm{~Hz}$. The cutoff frequency was set at $0.3 \mathrm{~Hz}$, because in newborn lambs, mean breathing rate is higher than $0.3 \mathrm{~Hz}$, with no significant differences among wake-sleep states (18), and the high-frequency peak is observed in the variability of cerebral perfusion pressure at frequencies higher than $0.3 \mathrm{~Hz}(17)$.

Time delays at the neural and effector levels (22) characterize the baroreceptor reflex. The highest positive correlation between HP and MAP occurred with HP following MAP by 1-2 s (Table 1). Time lags tended to be higher in modulus at low frequencies than on unfiltered data, the difference reaching significance in quiet sleep, emphasizing the contribution of high-frequency ventilatory fluctuations to HP and MAP variability in this state. In newborn lambs, phase-shift analysis in the frequency domain determined that heart rate preceded arterial pressure by $2.5 \mathrm{~s}$ at $0.1 \mathrm{~Hz}$ (24). Phase-shift analysis cannot unambiguously determine the temporal relationship between two signals: because heart rate is inversely related to $\mathrm{HP}$ and because half a period of an oscillation (i.e. $5 \mathrm{~s}$ at 0.1 $\mathrm{Hz})$ is needed to produce phase opposition, these data are 
compatible with HP following arterial pressure by $2.5 \mathrm{~s}$, in broad agreement with our findings. Accordingly, in adult humans, spontaneous fluctuations in HP follow those in arterial pressure at low frequencies by $1-2 \mathrm{~s}(23,25)$. Time lags at the positive peak of the HP versus MAP cross-correlation function may reflect the latency of the maximal sinus node response to a change in MAP (26) rather than the latency of the earliest sinus node response [cf. (25)].

In correspondence to the median time lags between changes in MAP and those in HP, we found that the HP versus MAP correlation coefficient significantly differed among states (Fig. $3)$, with the highest value attained in quiet sleep $(0.51 \pm 0.05)$. Data thus indicate that HP is coupled to previous MAP changes, the strength of the coupling being highest in quiet sleep, and suggest that the impact of the baroreflex on HP control is higher during quiet sleep than in the other states in newborn lambs. At low frequencies, the sleep-state-related difference in the coupling between HP and MAP was enhanced, the HP versus MAP correlation coefficient rising significantly to $0.61 \pm 0.04$ in quiet sleep (Fig. 4). Therefore, the significance of the difference in the HP versus MAP coupling among states was not dependent on high-frequency fluctuations in HP and MAP, which constitute the greatest fraction of ventilatory-induced cardiovascular variability in all wake-sleep states in newborn lambs (27). As ventilatory activity was not measured in the present study, we cannot exclude that minor low-frequency ventilatory fluctuations disrupted the coupling between HP and MAP, acting more in quiet wakefulness and in active sleep than in quiet sleep. However, this would indicate greater nonbaroreflex control of HP during quiet wakefulness and active sleep, in agreement with our conclusion. Our results are at variance with those of Gronlund et al. (24), who did not report significant sleep-related differences in the frequency domain coherence at $0.1 \mathrm{~Hz}$ between heart rate and arterial pressure in newborn lambs. Owing to the polysomnographic recordings that we performed, we were able to exclude microarousals and sleep-state transitions from the analysis, whereas such transient events may have been included in the analysis in the study of Gronlund et al. (24), who scored wake-sleep states on the basis of behavioral observation alone. Microarousals entail distinct polygraphic deviations from the pattern of the stable quiet sleep and are accompanied by transient cardiorespiratory events such as abrupt shifts in heart rate (28).

In stable quiet sleep, it is well known that cardiovascular variability is the lowest (1). Our study is novel in that we directly showed that the coupling between HP and MAP varies with the wake-sleep state and is the tightest in quiet sleep. This feature of cardiovascular regulation may partly underlie the low cardiovascular variability in quiet sleep. Our data in newborn lambs are in broad agreement with those previously obtained in adult rats, in which the correlation between HP and MAP at low frequencies was higher in quiet sleep than either in active sleep or in quiet wakefulness both in control conditions and when cardiovascular regulation was perturbed by acoustic stimuli presented during sleep (6). It is of interest that neither in adult rats (5) nor in newborn lambs (18) does the gain of the cardiac baroreflex change significantly among wake-sleep states. Differences in the correlation between HP and MAP therefore are suggestive of differences in the balance between the baroreceptor reflex and central commands in the regulation of heart rhythm.

Phasic increases (surges) in MAP during active sleep were initially concomitant with decreases in HP in lambs (Fig. 6). Because MAP surges in active sleep are primarily caused by increases in systemic vascular resistance in newborn lambs (19), the decrease in HP indicates that central autonomic commands to the heart and blood vessels phasically prevailed on baroreflex control in active sleep. Surges in heart rate during active sleep are mediated by a $\beta_{1}$-adrenergic mechanism in adult cats (29), but their beat-to-beat relationship with arterial pressure has not been reported. In our study, the HP response to an increase in centrally driven autonomic output to the heart was almost simultaneous with the MAP one, the lag lying within one cardiac period. However, HP started to increase after 5 cardiac cycles, to rise above control levels 10 cardiac cycles after the onset of the MAP surge (Fig. 6). The rise in HP is consistent with a delayed baroreflex response evoked by the increase in MAP, which became apparent after the central autonomic commands to the heart subsided. Such a complex regulatory arrangement, which causes biphasic changes in HP as a consequence of central autonomic commands to the heart and blood vessels, may constitute a challenge for heart rhythm regulation in pathologic conditions characterized by an increased risk for arrhythmias. Paradoxically, coronary vascular resistance increases during MAP surges in active sleep (19). Thus, the increase in myocardial oxygen demand caused by the rise in arterial pressure and heart rate at the surge onset may contribute to a condition of myocardial vulnerability in the late part of the night (30), when active sleep is prevalent.

In adult humans, sleep-related changes in the control of heart rhythm may help in the detection of autonomic derangements after myocardial infarction (31). In infancy, sudden infant death syndrome has been associated with derangements in autonomic cardiovascular control, which have been evidenced by standard tests (32) and may be linked to the pathophysiology of the syndrome (33). Such derangement may be most prominent during specific times of the night (14) and during specific sleep states (15). Knowledge of physiologic sleeprelated changes in cardiovascular regulation is necessary to detect their pathologic derangements. Our study shows a novel aspect of the physiologic sleep-related differences in autonomic cardiovascular control in newborn life.

\section{REFERENCES}

1. Franzini C 2000 Cardiovascular physiology: the peripheral circulation. In: Kryger MH, Roth T, Dement WC (eds) The Principles and Practice of Sleep Medicine. WB Saunders Company, Philadelphia, pp 193-203

2. Parmeggiani PL 1980 Behavioral phenomenology of sleep (somatic and vegetative) Experientia 36:6-11

3. DiCarlo SE, Bishop VS 2001 Central baroreflex resetting as a means of increasing and decreasing sympathetic outflow and arterial pressure. Ann NY Acad Sci 940:324-337

4. Schenberg LC, Vasquez EC, da Costa MB 1993 Cardiac baroreflex dynamics during the defence reaction in freely moving rats. Brain Res 621:50-58

5. Zoccoli G, Andreoli E, Bojic T, Cianci T, Franzini C, Predieri S, Lenzi P 2001 Central and baroreflex control of heart rate during the wake-sleep cycle in rat. Sleep 24:753-758

6. Silvani A, Bojic T, Cianci T, Franzini C, Lodi CA, Predieri S, Zoccoli G, Lenzi P 2003 Effects of acoustic stimulation on cardiovascular regulation during sleep. Sleep 26:201-205 
7. Manders WT, Pagani M, Vatner SF 1979 Depressed responsiveness to vasoconstrictor and dilator agents and baroreflex sensitivity in conscious, newborn lambs. Circulation 60:945-954

8. Walker AM, Horne RS, Bowes G, Berger P 1986 The circulation in sleep in newborn lambs. Aust Pediatr J 22(suppl 1):71-74

9. Siimes AS, Valimaki IA, Antila KJ, Julkunen MK, Metsala TH, Halkola LT, Sarajas HS 1990 Regulation of heart rate variability by the autonomic nervous system in neonatal lambs. Pediatr Res 27:383-391

10. Gaultier C 1995 Cardiorespiratory adaptation during sleep in infants and children. Pediatr Pulmonol 19:105-117

11. Patzak A, Lipke K, Orlow W, Mrowka R, Stauss H, Windt E, Persson PB, Schuber E 1996 Neonatal peculiarities of cardiorespiratory control. Am J Physiol 271:R1025R1032

12. Segar JL 1997 Ontogeny of the arterial and cardiopulmonary baroreflex during fetal and postnatal life. Am J Physiol 273:R457-R471

13. Challis RE, Kitney RI 1990 Biomedical signal processing (in four parts). Part 1. Time-domain methods. Med Biol Eng Comput 28:509-524

14. Franco P, Szliwowski H, Dramaix M, Kahn A 1998 Polysomnographic study of the autonomic nervous system in potential victims of sudden infant death syndrome. Clin Autonom Res 8:243-249

15. Schechtman VL, Harper RM, Kluge KA, Wilson AJ, Hoffman HJ, Southall DP 1988 Cardiac and respiratory patterns in normal infants and victims of the sudden infant death syndrome. Sleep 11:413-424

16. Grant DA, Davidson TL, Fewell JE 1995 Automated scoring of sleep in the neonatal lamb. Sleep 18:439-445

17. Silvani A, Bojic T, Franzini C, Lenzi P, Walker AM, Grant DA, Wild J, Zoccoli G 2004 Sleep-related changes in the regulation of cerebral blood flow in newborn lambs Sleep 27:36-41

18. Horne RS, De Preu ND, Berger PJ, Walker AM 1991 Arousal responses to hypertension in lambs: effect of sinoaortic denervation. Am J Physiol 260:H1283-H1289

19. Fewell JE 1993 Influence of sleep on systemic and coronary hemodynamics in lambs. J Dev Physiol 19:71-76

20. Sei H, Morita Y 1996 Acceleration of EEG theta wave precedes the phasic surge of arterial pressure during REM sleep in the rat. Neuroreport 7:3059-3062

21. de Swiet M, Fayers P, Shinebourne EA 1992 Blood pressure in first 10 years of life: the Brompton study. BMJ 304:23-26
22. Malpas SC 2002 Neural influences on cardiovascular variability: possibilities and pitfalls. Am J Physiol 282:H6-H20

23. Cevese A, Gulli G, Polati E, Gottin L, Grasso R 2001 Baroreflex and oscillation of heart period at $0.1 \mathrm{~Hz}$ studied by $\alpha$-blockade and cross-spectral analysis in healthy humans. J Physiol 531:235-244

24. Gronlund JU, Antila KJ, Siimes AS, Metsala T, Oja R, Tuominen J, Valimaki IA 1989 Beta-adrenergic control and inter-relationships between heart rate and blood pressure in neonatal lambs. Med Biol Eng Comput 27:163-170

25. Cooke WH, Hoag JB, Crossman AA, Kuusela TA, Tahvanainen KU, Eckberg DL 1999 Human responses to upright tilt: a window on central autonomic integration. J Physiol 517:617-628

26. Baskerville AL, Eckberg DL, Thompson MA 1979 Arterial pressure and pulse interval responses to repetitive carotid baroreceptor stimuli in man. J Physiol 297:61-71

27. Gronlund JU, Kalli ST, Siimes ASI, Sydanmaa M, Antila KJ, Valimaki IA 1991 Do $\beta$-adrenergic blockade and sleep state affect cardiorespiratory control in neonatal lambs? Multivariate autoregressive modeling approach. Pediatr Res 29:272-277

28. Quattrochi JJ, Shapiro J, Verrier RL, Hobson JA 2000 Transient cardiorespiratory events during NREM sleep: a feline model for human microarousals. J Sleep Res 9:185-191

29. Rowe K, Moreno R, Lau TR, Wallooppillai U, Nearing BD, Kocsis B, Quattrochi J, Hobson JA, Verrier RL 1999 Heart rate surges during REM sleep are associated with theta rhythm and PGO activity in cats. Am J Physiol 277:R843-R849

30. Patel DJ, Knight CJ, Holdright DR, Mulcahy D, Clarke D, Wright C, Purcell H, Fox KM 1997 Pathophysiology of transient myocardial ischemia in acute coronary syndromes. Characterization by continuous ST-segment monitoring. Circulation 95:1185-1192

31. Vanoli E, Adamson PB, Ba-Lin M, Pinna GD, Lazzara R, Orr WC 1995 Heart rate variability during specific sleep stages. A comparison of healthy subjects with patients after myocardial infarction. Circulation 91:1918-1922

32. Fox GP, Matthews TG 1989 Autonomic dysfunction at different ambient temperatures in infants at risk of sudden infant death syndrome. Lancet 2:1065-1067

33. Harper RM, Bandler R 1998 Finding the failure mechanism in sudden infant death syndrome. Nat Med 4:157-158 